\title{
Aktuelle Aspekte der Lepra-Therapie
}

\section{Bendick}

\section{Current Aspects of Leprosy Therapy}

\section{Zusammenfassung}

Die medikamentöse Therapie der Lepra hat in den letzten Jahrzehnten erhebliche Fortschritte gemacht. Vor dem Hintergrund der weltweiten Bemühungen um die Elimination der Erkrankung werden historische, gegenwärtige sowie neue und experimentelle Therapiestrategien erörtert und kritisch gewürdigt. Bedingungen für den künftigen Einsatz therapeutischer Regime sowie wesentliche Herausforderungen im Hinblick auf die medikamentöse Therapie der Lepra werden aufgezeigt.

\section{Abstract}

Drug therapy of leprosy has made great advances in the past decades. Regarding the worldwide efforts towards the elimination of the disease, historical, current and new, as well as experimental therapeutic strategies, are discussed and critically assessed. Conditions for the future application of therapeutic regimens, and essential challenges concerning the drug therapy of leprosy are elaborated.
Trotz herausragender Erfolge in der Bekämpfung der Lepra, welche vor allem seit 1981, dem Jahr der Einführung der Kombinationstherapie von Rifampicin, Dapson und Clofazimin (MDT), zu verzeichnen sind, spielen die Infektion und ihre Folgezustände nach wie vor eine bedeutende Rolle, vor allem in zahlreichen Ländern der Dritten Welt.

Vor dem Hintergrund der Bemühungen der Weltgesundheitsorganisation (WHO), Lepra bis zum Jahre 2005 weltweit zu eliminieren (definiert als Absenkung ihrer Prävalenz auf unter einen Fall pro 10.000 Einwohner), will der folgende Beitrag über den derzeitigen Stand der medikamentösen Therapie informieren.

Eindrucksvolle Erfolge der in den achtziger Jahren auf breiter Basis verabreichten MDT veranlassten im Mai 1991 die Teilnehmer der 44. Weltgesundheits-Konferenz, eine Elimination der Lepra für das Jahr 2000 ins Auge zu fassen [1]. Im Mai 2001 konnte umgerechnet auf die Weltbevölkerung - der erfolgreiche Vollzug verkündet werden [2]. Gleichwohl haben 15 Länder das Eliminationsziel noch nicht erreicht, wobei 83\% aller gemeldeten Leprapatienten in Brasilien, Madagaskar, Indien, Nepal, Myanmar und Indonesien beheimatet sind. Daher werden derzeit im Rahmen eines sog. Final Push Aktivitäten lanciert, die die Verwirklichung des Vorhabens auch auf nationaler Ebene für 2005 anvisieren [3]. Zur Erreichung des Ziels hat die WHO im November 1999 die Global Alliance for Leprosy Elimination ins Leben gerufen, deren wesentliches Anliegen es ist, die weltweit auf etwa 2,5 Millionen geschätzten unbehandelten Lepra-Fälle der Therapie zuzuführen. Auf die breite und kontroverse Diskussion über Voraussetzungen, Methoden und Ziele der WHO-propagierten Eliminationsbemühungen [4-6] soll im Rahmen dieses Artikels nicht eingegangen werden.

Die epidemiologische Situation weltweit Im Jahre 2002 ist in Abb. 1 dargestellt. 


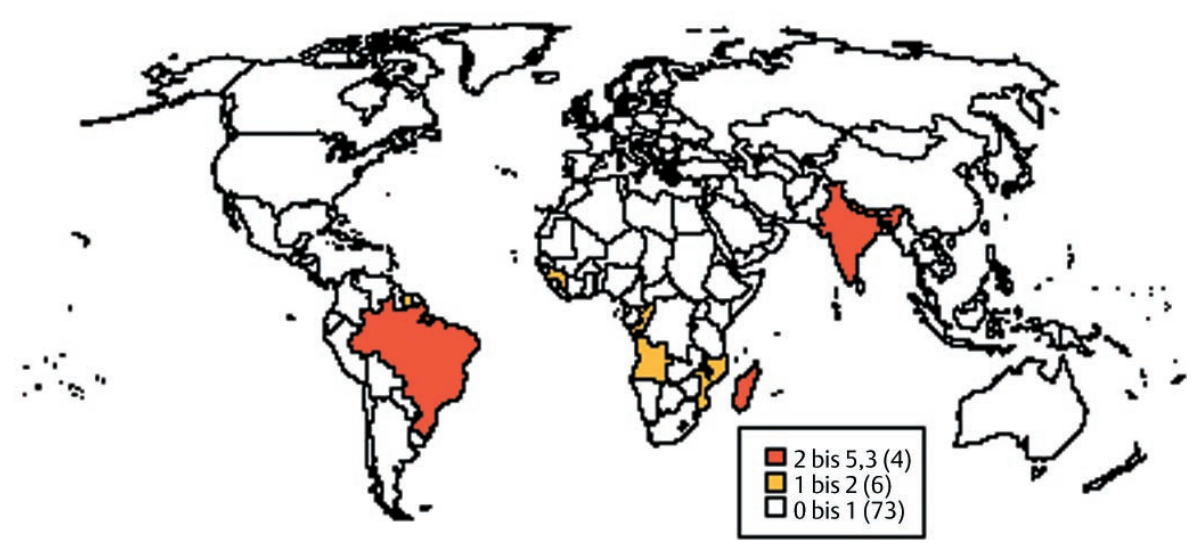

Abb. 1 Lepra-Prävalenz 2002. Anzahl der gemeldeten Fälle auf 10000 Einwohner, in Klammern die Anzahl der jeweils betroffenen Länder (Quelle: WHO).

Bis 1941 gab keine wirksame Therapie der Lepra und die Erkrankung galt als unheilbar. Das seit Jahrhunderten in Indien und China angewandte Chaulmoogra- bzw. Hydnocarpus-Öl war weitgehend wirkungslos [7]. 1941 führte Guy Faget ein DapsonDerivat $\left(\right.$ Promin $^{\circledR}$ ) ein, welches intravenös gegeben wurde [8]. Das bereits 1908 von Fromm und Wittmann in Freiburg zur Therapie der Tuberkulose synthetisierte Dapson [9] (4:4'-diaminodiphenylsulfon, DDS, DADPS) wurde zur Leprabehandlung erstmals 1946 von Cochrane in Indien intramuskulär [10], 1947 von Lowe in Nigeria oral [11] verabreicht. 1950 schließlich setzte sich die orale Gabe von Dapson als Standardtherapie weithin durch.

Bis 1981 wurde Dapson in der Behandlung der Lepra als Monotherapie gegeben. Bereits 1960 erkannte man jedoch, dass es nach Jahren ausschließlicher Dapson-Behandlung zur Resistenzentwicklung des Erregers Mycobakterium leprae kommen kann [12]. Patienten mit teils alarmierend hohen Raten primär resistenter Erreger von bis zu 40\% wurden erstmals in den siebziger Jahren beschrieben [13]. Basierend auf diesen Beobachtungen wurde einer Modifikation des bisherigen Therapieregimes dringend erforderlich. Die Kombination verschiedener antibiotisch wirksamer Substanzen bot - verbunden mit der Notwendigkeit einer in Hinblick auf Dosierung und Zeitdauer adäquaten Einnahme - die beste Aussicht, die Resistenzbildung einzudämmen und damit eine Ausbreitung therapieresistenter M. leprae-Stämme weitgehend zu unterbinden. Dementsprechend befürwortete die WHO Study Group im Jahre 1982 [14] eine Kombinationstherapie aus Dapson und Rifampicin für Patienten mit paucibazillärer (PB, d. h. Lepra-Formen mit im Ausstrich nicht nachweisbaren Bakterien) sowie Dapson, Rifampicin und Clofazimin für Patienten mit multibazillärer Lepra (MB, d.h. Lepra-Formen mit im Ausstrich nachweisbarer Bakterienlast). Seit 2000 gibt die WHO folgende Empfehlung [15] (Tab.1).

Die Medikamente werden in hitze- und feuchtigkeitsbeständigen Blister-Packs (Abb. 2) ausgegeben, welche es dem Patienten erleichtern, die korrekte tägliche Dosis zu sich zu nehmen [16]. Auch Sehbehinderte oder Analphabeten sind in der Regel ohne weiteres in der Lage, mit der so abgepackten Arznei umzugehen, welche in voller Dosierung von Anfang bis Ende des Therapiezeitraumes verabreicht werden sollte. Auch in der Schwangerschaft oder in Perioden von Typ I- oder II-Reaktionen ${ }^{1}$ ist ein Aussetzen der Therapie nicht indiziert. Dies ist außerordentlich wichtig, um eine weitere Resistenzbildung zu unterbinden (weitreichende Resistenz gegen Rifampicin beispielsweise wür-
Tab. 1 Seit 2000 von der WHO empfohlenes Therapieregime für pauci- und multibazilläre Lepra bei Erwachsenen und Kindern

\begin{tabular}{|c|c|c|}
\hline \multicolumn{3}{|c|}{ Erwachsene Patienten } \\
\hline Art der Lepra & $P B$ & MB \\
\hline $\begin{array}{l}\text { Anzahl der } \\
\text { Läsionen }\end{array}$ & $1-5$ & $>5$ \\
\hline $\begin{array}{l}\text { bakterieller } \\
\text { Index (Ridley- } \\
\text { Skala [57]) }\end{array}$ & negativ & positiv \\
\hline \multirow[t]{2}{*}{ Regime } & täglich: Dapson 100 mg & $\begin{array}{l}\text { täglich: Dapson } 100 \text { mg, } \\
\text { Clofazimin } 50 \text { mg }\end{array}$ \\
\hline & $\begin{array}{l}\text { einmal monatlich: } \\
\text { Rifampicin } 600 \text { mg }\end{array}$ & $\begin{array}{l}\text { einmal monatlich: Rifampicin } \\
600 \mathrm{mg} \text {, Clofazimin } 300 \mathrm{mg}\end{array}$ \\
\hline Therapiedauer & 6 Monate & 12 Monate \\
\hline
\end{tabular}

Monatliche Dosen sind unter Supervision zu verabreichen.

Kinder mit PB Lepra

$\begin{array}{lll}\text { Altersgruppe tägliche Dapson- } & \text { monatliche Rifampicin- } \\ \text { Dosis } & \text { Dosis }\end{array}$

\begin{tabular}{|c|c|c|}
\hline 10-14 Jahre & $50 \mathrm{mg}$ & $450 \mathrm{mg}$ \\
\hline 15-18 Jahre & $100 \mathrm{mg}$ & $600 \mathrm{mg}$ \\
\hline Therapiedauer & \multicolumn{2}{|c|}{6 Monate } \\
\hline \multicolumn{3}{|c|}{ Monatliche Dosen sind unter Supervision zu verabreichen. } \\
\hline \multicolumn{3}{|c|}{ Kinder < 10 Jahre erhalten an ihr Körpergewicht angepasste Dosen. } \\
\hline
\end{tabular}

\begin{tabular}{|c|c|c|c|c|}
\hline \multicolumn{5}{|c|}{ Kinder mit MB Lepra } \\
\hline Altersgruppe & $\begin{array}{l}\text { tägliche } \\
\text { Dapson- } \\
\text { Dosis }\end{array}$ & $\begin{array}{l}\text { monatliche } \\
\text { Rifampicin- } \\
\text { Dosis }\end{array}$ & $\begin{array}{l}\text { tägliche } \\
\text { Clofazimin- } \\
\text { Dosis }\end{array}$ & $\begin{array}{l}\text { monatliche } \\
\text { Clofazimin- } \\
\text { Dosis }\end{array}$ \\
\hline 10-14 Jahre & $50 \mathrm{mg}$ & $450 \mathrm{mg}$ & $\begin{array}{l}50 \mathrm{mg} \\
\text { jeden 2.Tag }\end{array}$ & $150 \mathrm{mg}$ \\
\hline 15-18 Jahre & $100 \mathrm{mg}$ & $600 \mathrm{mg}$ & $50 \mathrm{mg}$ & $300 \mathrm{mg}$ \\
\hline Therapiedauer & \multicolumn{4}{|c|}{12 Monate } \\
\hline \multicolumn{5}{|c|}{ Monatliche Dosen sind unter Supervision zu verabreichen. } \\
\hline \multicolumn{5}{|c|}{ Kinder < 10 Jahre erhalten an ihr Körpergewicht angepasste Dosen. } \\
\hline
\end{tabular}

${ }^{1}$ Exazerbationen des ansonsten hoch chronischen Krankheitsverlaufes der Lepra werden als Reaktionen bezeichnet. Man unterscheidet die Typ I-Reaktion, welche durch einen - oftmals therapieinduzierten - Anstieg der zellvermittelten Immunität verursacht wird, von der Typ II-Reaktion (Erythema nodosum leprosum [ENL]), die man als ein durch Präzipitation von $\mathrm{Ag} / \mathrm{Ak}$-Komplexen verursachtes Immunkomplex-Syndrom auffasst. Typ I-Reaktionen kommen bei BT, BB- und BL-, selten auch bei TT-Fällen vor, Typ II-Reaktionen sind in aller Regel auf LL-, gelegentlich auch BL-Fälle beschränkt. 


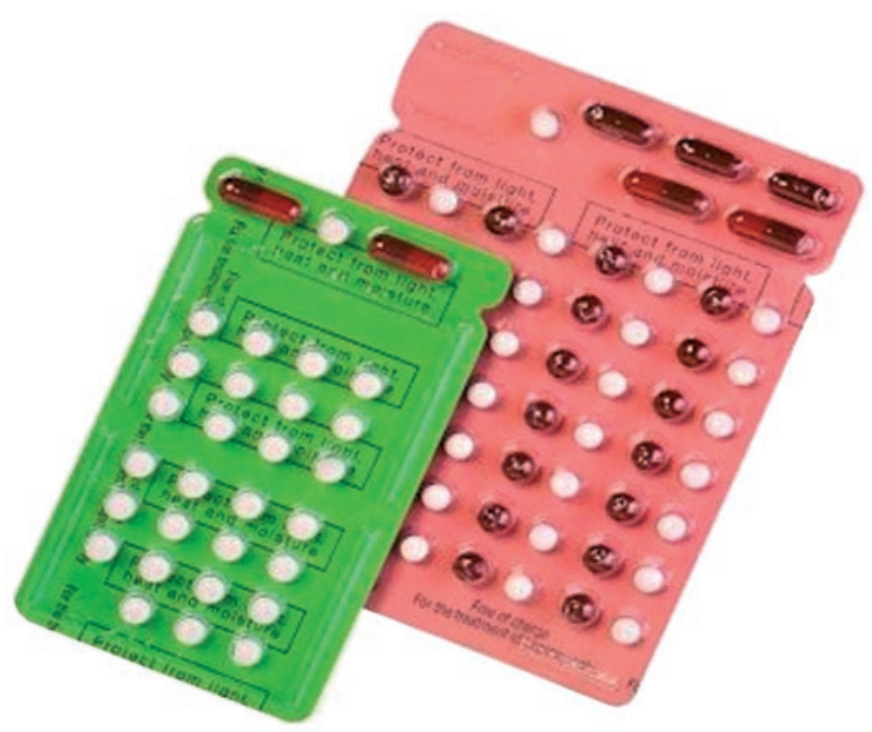

Abb. 2 Rechts ein Blister-Pack für MB-, links für PB-Lepra (Erwachsene).

de das gesamte gegenwärtige Konzept der Lepratherapie in Frage stellen). Die supervidierte Verabreichung dient dem Zweck, dem Patienten seine monatliche Dosis auszuhändigen und ihn so an sein Gesundheitszentrum zu binden. Dies ermöglicht regelmäßigen Kontakt, um nach dem Fortgang der Therapie und eventuellen Komplikationen zu fragen [17]. In sehr unterentwickelten Ländern soll so auch der Verkauf des relativ teuren Rifampicin aus dem Blister-Pack durch manche Patienten unterbunden werden. Die Kooperation von WHO, Pharma-Industrie und anderen Sponsoren erlaubt es der Global Alliance for Elimination of Leprosy, die notwendigen Medikamente weltweit unentgeltlich zur Verfügung zu stellen.

Um die klinische Erscheinungsvielfalt der Lepra zu systematisieren, entwickelten Ridley und Jopling 1966 eine an der zellulären Immunität des betroffenen Individuums orientierte Klassifikation [18], welche fünf Manifestationsformen der Erkrankung unterscheidet: tuberkuloide (TT) (Abb.3), borderline-tuberkuloide (BT), mid-borderline (BB) (Abb.4), borderline-lepromatöse (BL) und lepromatöse (LL) (Abb.5) Lepra. Außerhalb dieses Spektrums stehen die durch das Auftreten einer bzw. mehrerer unspezifischer Läsionen mit oder ohne Einschränkung der Sensitivität charakterisierte indeterminierte Lepra (I) sowie die - vorwiegend in Indien vorkommende - primär neuritische Lepra, welche durch den ausschließlichen Befall von Nerven gekennzeichnet ist. Histoide Lepra und die hauptsächlich auf Mittelund Südamerika beschränkte Lucio-Lepra stellen klinisch und histologisch fassbare Varianten der Lepra lepromatosa dar.

Im klinischen Alltag und vor allem und im Hinblick auf die korrekte Therapieentscheidung unter Feldbedingungen ist das Ridley-Jopling-Schema jedoch oftmals zu differenziert. Hierfür empfiehlt sich eher die WHO-Klassifikation [19], welche BB-, BL- und LL-Fälle als MB-Lepra, I-, TT- und die Mehrzahl der BTFälle als PB-Lepra kennzeichnet (kann jedoch bei I-, TT- und BTFällen im Ausstrich M. leprae nachgewiesen werden, gelten auch diese als MB und müssen entsprechend behandelt werden). Noch weiter vereinfacht und nicht angewiesen auf Laborunter- suchungen ist die Methode, Patienten mit weniger als fünf Läsionen als paucibazillär, solche mit mehr als fünf Läsionen als multibazillär zu bezeichnen (siehe auch Tab.1).

Die zur Verfügung stehenden Medikamenten lassen sich wie folgt charakterisieren:

Dapson wird nach oraler Aufnahme fast vollständig resorbiert. Die Substanz ist vorwiegend bakteriostatisch, weniger bakterizid wirksam. Vier Stunden nach Einnahme ist der maximale Wirkspiegel, welcher die minimale Hemmkonzentration gegen M.leprae um das 500 fache übersteigt, erreicht. Nebenwirkungen sind unter Einnahme von Dapson nicht ungewöhnlich [20]. So wurde über Unwohlsein, Fieber, Leukopenie, Methaemoglobinämie sowie Fälle von Nephritis, peripherer Neuritis, Hepatitis und akuten psychotischen Episoden berichtet. Seitens der Haut ist auf fixe und generalisierte Arzneiexantheme bis hin zu Erythrodermien und toxisch epidermaler Nekrolyse (TEN) zu achten. Das in der vierten bis sechsten Einnahmewoche auftretende, mit Fieber, Erythrodermie, Nephritis und Hepatitis einhergehende und potentiell fatale „Dapson-Unverträglichkeits-Syndrom“ ist ein sehr seltenes Ereignis. Wesentlichste Nebenwirkung ist die Manifestation einer hämolytischen Anämie, vor allem bei Patienten mit Glucose-6-phosphat-dehydrogenase (G6PD)-Mangel [21].

Das bakterizide Rifampicin ist in der Standard-MDT die Substanz mit der höchsten Wirksamkeit gegen M. leprae. Eine Dosis von $600 \mathrm{mg}$ ist in der Lage, 99,9\% der Bakterienlast eines Individuums zu eliminieren; Patienten werden so innerhalb weniger Tage nicht-infektiös. Rifampicin wird meist gut vertragen. Schwindel und Unwohlsein sowie Arzneiexantheme und gastrointestinale Störungen kommen vor. Wesentlich seltener sind Fälle von Hepatitis, Porphyria cutanea tarda, Pemphigus vulgaris und Stevens-Johnson-Syndrom. Häufig und ungefährlich ist die durch Abbauprodukte der Substanz hervorgerufene Rosa- bis Rotfärbung von Körperflüssigkeiten. Vor allem in den siebziger Jahren wurde sehr kontrovers über Fragen der Dosierung, des Intervalls zwischen zwei Gaben und der erforderlichen Therapiedauer diskutiert [22-24].

Das erstmals 1962 von Browne und Hogerzeil [25] in der Therapie der Lepra angewandte Clofazimin ist im Wesentlichen bakteriostatisch und weniger bakterizid wirksam. Im Gegensatz zu Dapson und Rifampicin verfügt die Substanz über antiinflammatorische Eigenschaften [26], welche sie auch in der Therapie der Typ II-Reaktion (Erythema nodosum leprosum [ENL]) einsetzbar macht. Das Spektrum an Nebenwirkungen umfasst eine braune bis blaugraue Hyperpigmentierung der Haut, vor allem im Bereich UV-exponierter Areale (was immer wieder Patienten zum Therapieabbruch veranlasst), darüber hinaus können Arzneiexantheme, Xerosis und erhöhte Lichtempfindlichkeit auftreten. Auch über abdominale Schmerzen und Diarrhöen wurde berichtet; alle diese Symptome sind dosisabhängig und daher bei der Therapie des ENL stärker ausgeprägt [27] als bei der Standardbehandlung der MB-Lepra.

Resistenzen sind sowohl für Dapson wie auch für Rifampicin $[28,29]$, nicht jedoch bei Clofazimin beschrieben. Insgesamt scheinen sie eine untergeordnete Rolle bei Patienten unter MDT 


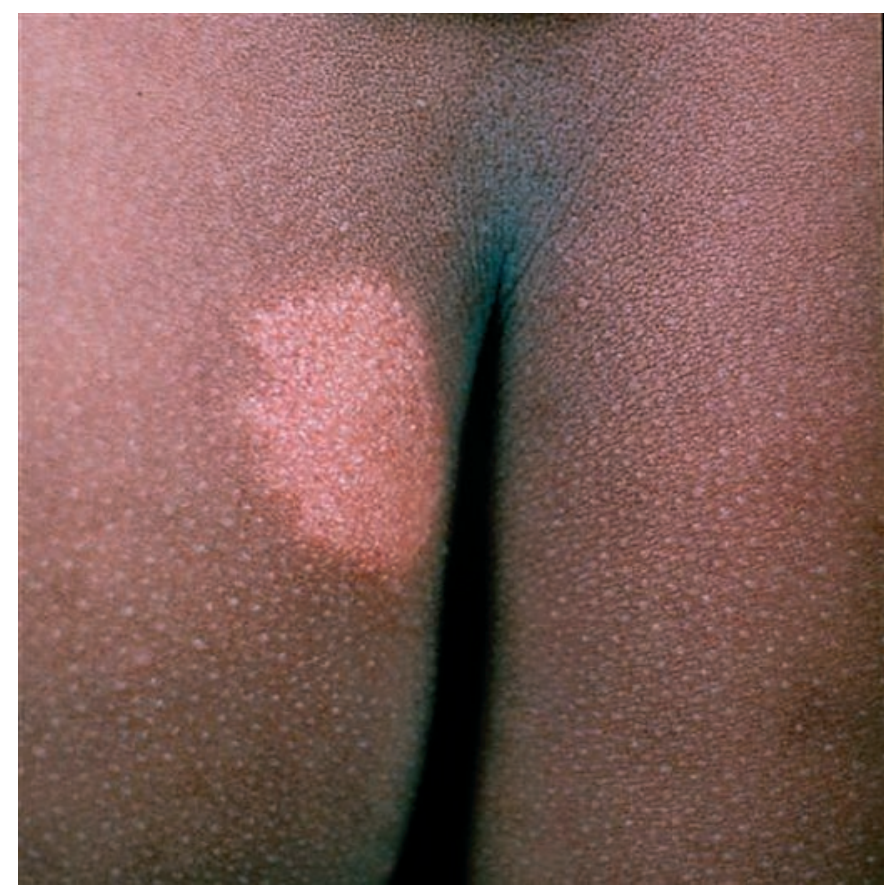

Abb. 3 TT-Lepra, @ Sasakawa Memorial Health Foundation.

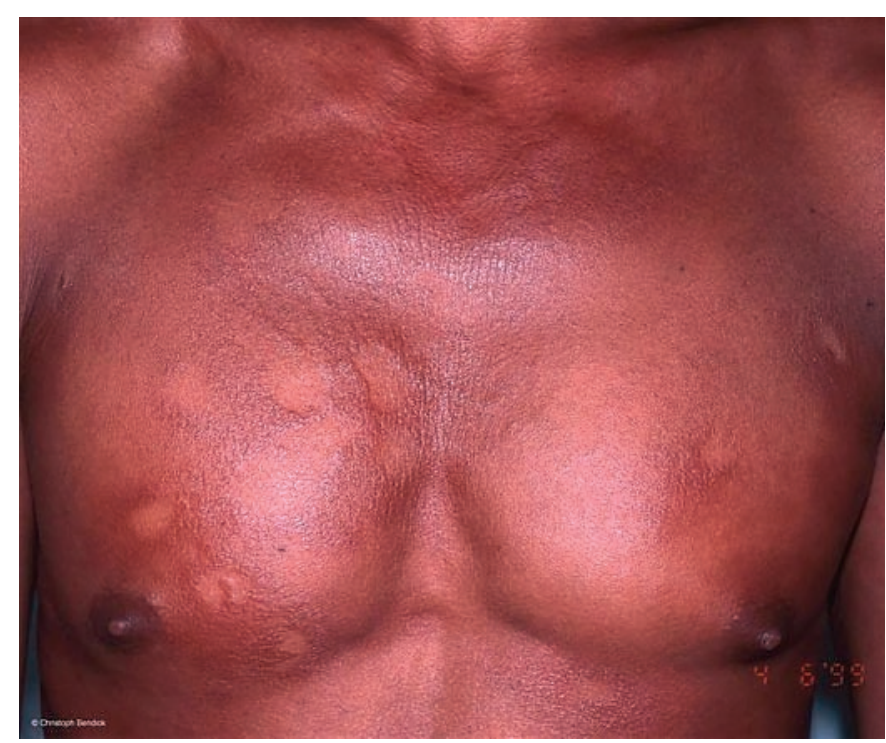

Abb. 4 BB-Lepra.

zu spielen, rezente zuverlässige Untersuchungen fehlen jedoch weitgehend, da seit etwa zehn Jahren Patienten nach Beendigung ihres MDT-Zyklus nicht mehr routinemäßig überwacht werden [30].

Zusätzlich kommen eine Reihe weiterer Substanzen in Betracht sei es als Ausweichmedikation bei Unverträglichkeiten oder Allergien, sei es im Rahmen von Tierversuchen oder neuer und experimenteller Therapieschemata.

Rifabutin aus der Familie der Ansamycine stellt eine Alternative zum Rifampicin dar [31]. Es hat starke bakterizide Wirkung, seine Verträglichkeit ist gut. Auch Rifapentin wird derzeit getestet

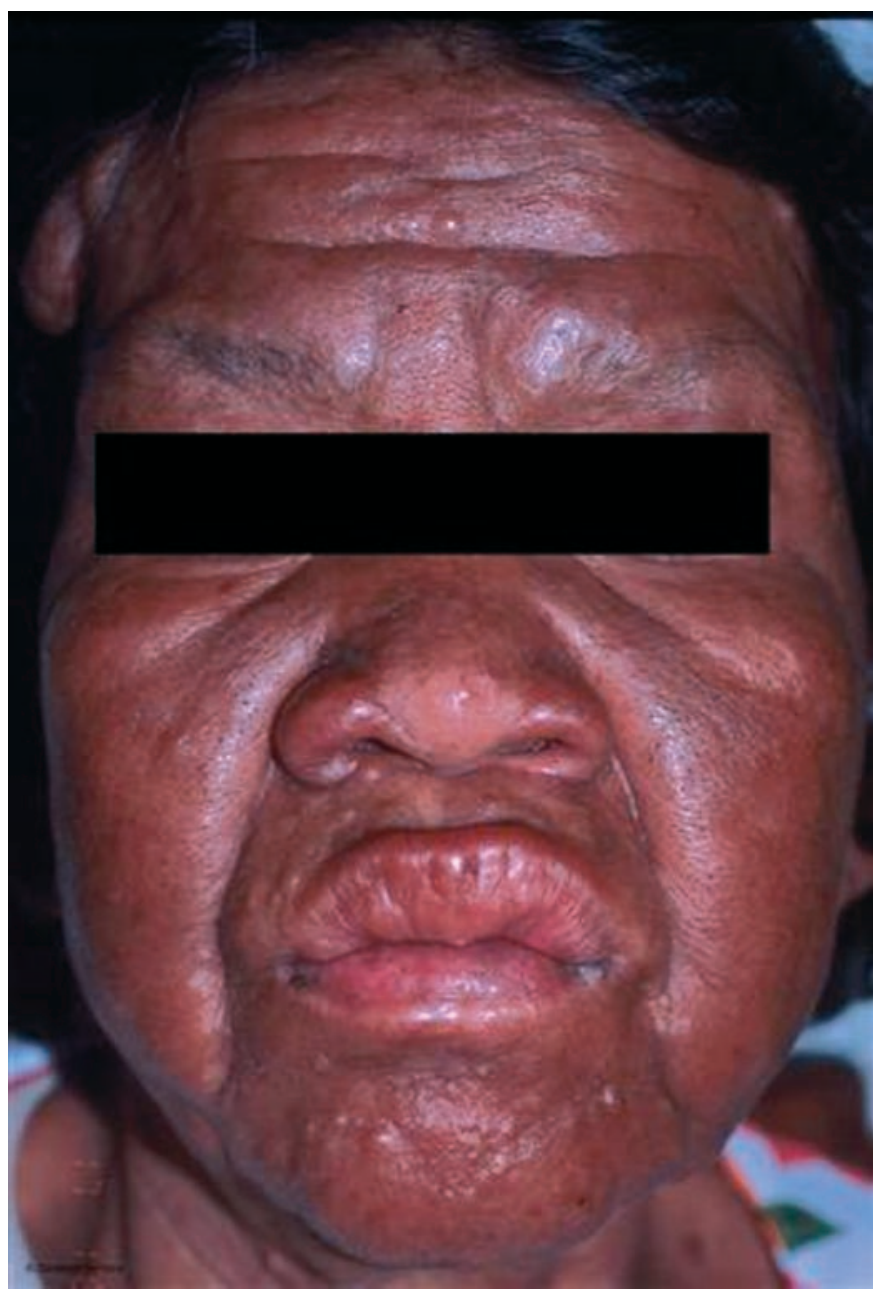

Abb. 5 Patientin aus Kambodscha mit LL-Lepra.

[32], seine antilepröse Potenz übersteigt die von Rifampicin deutlich [33].

Das Makrolid Clarithromycin zeigte sowohl als Monotherapeutikum [34] wie auch in der Kombination mit anderen Substanzen [35] antilepröse Wirksamkeit.

Unter den Fluorochinolonen steht Ofloxazin im Vordergrund. Bei einer Bioverfügbarkeit von 98\% demonstriert es im Tierversuch ausgesprochen hohe antilepröse Aktivität [36]. Das Spektrum der Nebenwirkungen umfasst Arzneiexantheme, Vaskulitiden, psychische Störungen, Sehstörungen und Haematurie. Ofloxazin wird im Rahmen der Einmalbehandlung von PB-Fällen mit nur einer Läsion angewendet (s. u.). Außerdem könnte sich das Medikament als Alternative bei Rifampicin-resistenter Lepra erweisen. Die Fluorochinolone Moxifloxazin [37], Levofloxazin [38] und Sitafloxazin [39] sind im Tierversuch stärker wirksam als Rifampicin. Auch Pefloxazin [40] und Sparfloxazin [41] wurden erfolgreich auf ihre antilepröse Potenz untersucht.

Unter den Tetrazyklinen ist Minozyklin [42] hervorzuheben. Es ist bakterizid und in seiner Wirksamkeit dem Ofloxazin vergleichbar, d.h. stärker wirksam als Dapson und Clofazimin, jedoch deutlich schwächer als Rifampicin und Rifabutin. Derzeit laufen Studien zur Wirksamkeit einer einmaligen monatlichen 


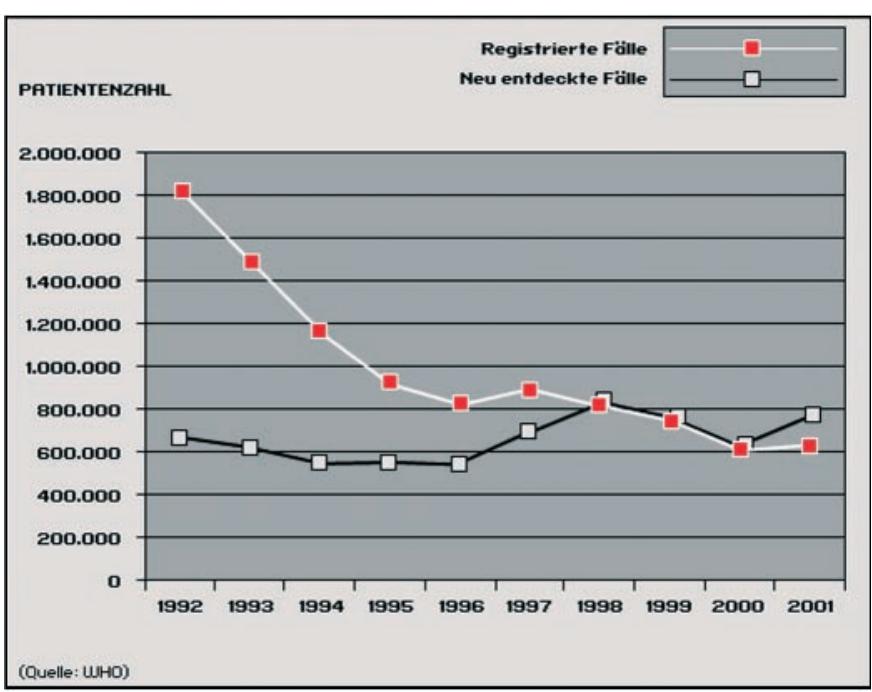

Abb. 6 Zahl der registrierten Leprapatienten und der neu entdeckten Fälle (registrierte „Prävalenz“ und „Inzidenz“) in absoluten Zahlen $1992-2001$.

Gabe von $600 \mathrm{mg}$ Rifampicin, $400 \mathrm{mg}$ Ofloxazin und $100 \mathrm{mg} \mathrm{Mi}-$ nozyklin über sechs (PB-) bzw. zwölf (MB-Lepra) Monate.

Einen Sonderfall stellt die PB-Lepra mit nur einer Läsion dar. Sie repräsentiert z. B. in Indien bis zu $60 \%$ aller neu diagnostizierten Fälle, und man hat vorgeschlagen, sie als eigene Entität einzuordnen [43]. Die Ergebnisse einer multizentrischen Doppelblindstudie an 1483 indischen Patienten scheinen die Wirksamkeit der einmaligen Gabe von $600 \mathrm{mg}$ Rifampicin, $400 \mathrm{mg}$ Ofloxazin und $100 \mathrm{mg}$ Minozyklin (ROM) bei single-lesion PB-Lepra zu rechtfertigen [44,45]. Gleichwohl ist die Standardtherapie (Dapson und Rifampicin für sechs Monate) diesem Regime in der Wirksamkeit leicht überlegen, zudem wurde die Qualität der o.a. Studie verschiedentlich in Zweifel gezogen [46]. Das singlelesion-single-dose-Konzept hat sich daher bislang nicht auf breiter Front durchgesetzt. Es kommt hauptsächlich in Indien, Bangladesch und Brasilien zur Anwendung [47].

Die medikamentöse Behandlung der Lepra hat sich in den vergangenen Jahrzehnten bedeutend verbessert. Welche zukünftigen Aussichten und Herausforderungen bietet die Chemotherapie?

- Die optimale MDT-Dauer bei MB-Lepra ist noch nicht abschließend geklärt. Während die sechsmonatige Therapiedauer für PB-Fälle gesichert ist, besteht trotz umfangreicher Erfahrungen für MB-Fälle noch kein endgültiger Konsens bezüglich der adäquaten Behandlungszeit und damit verbundener Rezidivraten. Ursprünglich auf eine Behandlungsdauer von mindestens 24 Monaten bzw. so lange, bis im Ausstrich $M$. leprae nicht mehr nachgewiesen werden konnte, angesetzt [48], empfahl die WHO 1994 eine für alle MB-Fälle einheitliche Therapiedauer von 24 Monaten [49]. 1998 ging die Empfehlung auf eine zwölfmonatige Behandlungsphase [50], und seit 2002 schließlich propagiert die WHO ein für MB- und PBLepra zeitlich einheitliches sechsmonatiges Therapiekonzept [51]. Solange die Rezidivquote bei der derzeit weltweit am häufigsten eingehaltenen Behandlungsdauer von zwölf Monaten bei MB-Fällen noch nicht zuverlässig abgeschätzt werden kann, muss eine erneute Verkürzung der Behandlungs- zeit (so wünschenswert dies aus organisatorischer Sicht auch sein mag) als verfrüht bezeichnet werden [52].

- In den letzten Jahren wurde aus operationalen Gründen auch die „begleitete MDT“ befürwortet [53]. Sie propagiert, dem Patienten zu Therapiebeginn die volle Medikamentendosis für sechs bzw. zwölf Monate auszuhändigen und ihn - mit Ausnahme des Auftretens akuter Komplikationen oder Probleme - erst am Ende der Therapieperiode wieder zu kontrollieren. Dieses Regime birgt zahlreiche Unsicherheiten hinsichtlich der überaus wichtigen Patientencompliance, ist daher umstritten [54] und hat sich bislang nicht durchsetzen können.

- Unbedingt notwendig ist eine sorgsame, mindestens fünfjährige, Nachbeobachtung von MB-Fällen, welche zwölf Monate lang behandelt wurden, sowie von PB-Fällen, die eine einmalige Dosis ROM erhalten haben - nur dies erlaubt die zuverlässige Diagnose von Rezidiven und den Vergleich mit der Rezidivhäufigkeit von Patienten, welche 24 bzw. sechs Monate konventionell therapiert wurden.

- Weitere Arbeit an der Identifizierung neuer antileprös wirksamer Medikamente bzw. weitere Untersuchung viel versprechender Substanzen ist gerechtfertigt. Obwohl dies vor dem Hintergrund gesehen werden muss, dass die gegenwärtig empfohlene MDT seit mehr als 20 Jahren angewendet wird, gute Resultat erbringt und dem Patienten kostenlos zur Verfügung gestellt wird, können sich gleichwohl Situationen ergeben, die den Einsatz neuer Präparate erforderlich machen: etwa bei allergischen oder Unverträglichkeitsreaktionen auf Komponenten der Standardtherapie, Resistenzentwicklung oder schlechtes Ansprechen auf die konventionelle MDT [55]. Auch eine weitere Reduzierung der Therapiedauer könnte die Notwendigkeit inhaltlich veränderter Behandlungsschemata mit sich bringen.

- Nachdem sowohl das menschliche wie auch das Genom von M. leprae entschlüsselt wurden, werden künftig vor allem im Hinblick auf die Beurteilung der Entwicklung von Resistenzen molekularbiologische Verfahren eine wesentliche Rolle spielen [56].

- Die Lepra-Inzidenz (Abb. 6) ist anhaltend hoch, und zwar auch in solchen Gegenden, in welchen die MDT-Anwendung intensiv propagiert wurde. Ob dies mit einer unzureichenden medikamentösen Abdeckung, bislang unerkannten nichtmenschlichen Überträgern, außergewöhnlich langen Inkubationszeiten oder partiell ineffizienten therapeutischen Regimen erklärt werden kann, bedarf der weiteren intensiven Untersuchung.

Auch wenn es gelingen sollte, Lepra zu eliminieren, bleiben zahlreiche Probleme auf absehbare Zeit bestehen: das kontinuierliche Auftreten von Leprafällen, die mitunter schwierige Therapie von Komplikationen, die - teils lebenslange - Therapiebedürftigkeit behinderter Patienten, die permanente Notwendigkeit von Aufklärungskampagnen, die fortgesetzte Ausbildung qualifizierter Gesundheitsarbeiter, der anhaltende Kampf gegen die Stigmatisierung der Betroffenen.

Viele Schlachten gegen die seit Jahrhunderten gefürchtete Erkrankung sind erfolgreich geschlagen - gewonnen ist der Kampf noch lange nicht. 
Herrn Professor Dr. Peter Stingl, Steingaden, danke ich für die Durchsicht des Manuskriptes.

\section{Literatur}

${ }^{1}$ World Health Assembly. Leprosy resolution 44.9. Forty-fourth World Health Assembly, 13. 5.1991

2 World Health Assembly. Press release World Health Assembly 54/2, 16. 5.2001

${ }^{3}$ World Health Organization. The final push strategy to eliminate leprosy as a public health problem. Questions and answers. 2.Aufl Genf: World Health Organization, 2003

${ }^{4}$ Lockwood DN. Leprosy elimination - a virtual phenomenon or a reality? Brit Med J 2002; 324 (7352): 1516-1518

${ }^{5}$ Stearns AT. Leprosy: a problem solved by 2000. Lepr Rev 2002; 73 (3): $215-224$

${ }^{6}$ Feenstra P. „Elimination“ of leprosy and the need to sustain leprosy services, expectations, predictions and reality. Int J Lepr Other Mycobact Dis 2003; 71 (3): 248-256

${ }^{7}$ Yawalkar SJ. Leprosy for Medical Practitioners and Paramedical Workers. 7. Auflage Basel: Novartis Stiftung, 2002

${ }^{8}$ Faget GH, Pogge RC, Johansen FA, Dinan JF, Prejean BM, Eccles CG. The promin treatment of leprosy - a progress report. Pub Health Rep 1943; 58: $1729-1741$

${ }^{9}$ Fromm E, Wittmann J. Derivate des p-Nitrophenols. Berichte Deutsch Chem Ges 1908; 41: 2264-2273

10 Cochrane R, Ramanujam H, Paul H et al. Two-and-a-half years experimental work on the sulphone group of drugs. Lepr Rev 1949; 20: 4-64

${ }^{11}$ Lowe J. The late results of sulphone treatment of leprosy in East Nigeria. Lepr Rev 1954; 25 (3): 13-124

${ }^{12}$ Pettit JHS, Rees RJW. Sulphone resistance in leprosy. An experimental and clinical study. Lancet 1964; II: 673-674

${ }^{13}$ Pearson JMH, Haile GS, Rees RJW. Primary dapsone-resistant leprosy. Lepr Rev 1977; 48 (2): 129-132

${ }^{14}$ WHO-Study Group. Chemotherapy of leprosy for control programmes. Tech Rep Ser 675 Genf: World Health Organization, 1982

${ }^{15}$ World Health Organization. Guide to Eliminate Leprosy as a Public Health Problem. WHO/CDS/CPE/CEE/2000.14 Genf: World Health Organization, 2000

${ }^{16}$ Georgiev GD, McDougall AC. Blister calendar packs - potential for the improvement in supply and utilization of multiple drug therapy in leprosy control programmes. Int J Lepr 1988; 56 (4): 603 - 610

${ }^{17}$ Huikeshoven H. Patient compliance in leprosy control: a necessity in old and new regimens. Int J Lepr 1985; 53 (3): $474-480$

18 Ridley DS, Jopling WH. Classification of leprosy according to immunity. A five-group system. Int J Lepr Other Mycobact Dis 1966; 34 (3): $255-273$

${ }^{19}$ WHO Expert Committee on Leprosy. 6th Report. Tech Rep Ser 768 Genf: World Health Organization, 1988

20 Goulart IM, Arbex GL, Carneiro MH, Rodrigues MS, Gadia R. [Adverse effects of multidrug therapy in leprosy patients: a five-year survey at a Health Center of the Federal University of Uberlandia]. Rec Soc Bras Med Trop 2002; 35 (5): 453 - 460 (portugiesisch)

${ }^{21}$ Editorial. Adverse reactions to dapsone Lancet 1981; II: 184- 185

22 Rees RJW, Pearson JMH, Waters MFR. Experimental and clinical studies of rifampicin in treatment of leprosy. Brit Med J 1970; 1: 89-x

${ }^{23}$ Leiker DL, Kamp H. First results of treatment of leprosy with Rifadin. Lepr Rev 1970; 41 (1): 25 - 30

${ }^{24}$ Languillon J, Yawalkar SJ, McDougall AC. Therapeutic effect of adding rimactane (rifampicin) 450 milligrams daily or 1200 milligrams once monthly in a single dose to dapsone 50 milligrams daily in patients with lepromatous leprosy. Int J Lepr 1979; 47 (1): 37-43

25 Browne SG, Hogerzeil LM. „B 663 “ in the treatment of leprosy. Preliminary report of a pilot trial. Lepr Rev 1962; 33: 6-10

${ }^{26}$ Waters MFR. G 30320 or B 663 - Lampren (Geigy). Lepr Rev 1969; 40 (1): $21-47$

27 Zimmerli-Ning M, Vischer W. A retrospective survey of the tolerability of clofazimine in patients with bacilliferous leprosy. Abstract III/137(A) New Delhi: XII. International Leprosy Congress, 1984

${ }^{28}$ Jacobson RR, Hastings RC. Rifampicin resistant leprosy. Lancet 1976; II: $1304-1305$
${ }^{29}$ Grosset JH, Guelpa-Lauras CC, Bobin P, Brucker G, Cartel JL, ConstantDesportes M, Flageul B, Frederic M, Guillaume JC, Millan J. Study of 39 documented relapses of multibacillary leprosy after treatment with rifampicin. Int J Lepr 1989; 57 (3): 607-614

${ }^{30}$ WHO Study Group. Chemotherapy of leprosy. Tech Rep Ser 874 Genf: World Health Organization, 1998

${ }^{31}$ Yoder LJ, Jacobson RR, Hastings RC. The activity of rifabutin against Mycobacterium leprae. Lepr Rev 1991; 62 (3): 280 - 287

$32 \mathrm{Ji} \mathrm{B}$, Grosset J. Combination of rifapentine-moxifloxacin-minocycline (PMM) for the treatment of leprosy. Lepr Rev 2000; 71 (Suppl.): S 81 - S 87

${ }^{33}$ Grosset JH. Newer drugs in leprosy. Int J Lepr Other Mycobakt Dis 2001; 69 (Suppl.): S14-S18

${ }^{34}$ Gelber RH. Successful treatment of a lepromatous patient with clarithromycin. Int J Lepr Other Mycobact Dis 1995; 63 (1): 113-115

35 Ji B, Jamet P, Perani EG, Sow S, Lienhardt C, Petinon C, Grosset JH. Bactericidal activity of single dose of clarithromycin plus minocycline, with or without ofloxacin, against Mycobacterium leprae in patients. Antimicrob Agents Chemother 1996; 40 (9): 2137-2141

36 Ji B, Grosset JH. Ofloxacin for the treatment of leprosy. Acta Lepr 1991; 7 (4): $321-326$

37 Consigny S, Bentoucha A, Bonnafous P, Grosset J, Ji B. Bactericidal activities of HMR 3647, moxifloxacin and rifapentine against Mycobakterium leprae in mice. Antimicrob Agents Chemother 2000; 44 (10): $2919-2921$

${ }^{38}$ Nakashima M, Uematsu T, Kanamaru M, Okazaki O, Hakusui H. Phase I study of levofloxacin,(s)-(-)-ofloxacin. Jpn J Clin Pharmacol Ther 1992; 23: $515-521$

${ }^{39}$ Dhople AM, Namba K. In vivo susceptibility of Mycobacterium leprae to sitafloxacin (DU-6859a), either singly or in combination with rifampicin analogues. Int J Antimicrob Agents 2003; 21 (3): 251 - 255

40 Bharti R. Pefloxacin in leprosy. Indian J Lepr 1994; 66 (4): 443 - 448

${ }^{41}$ Chan GP, Garcia-Ignacio BY, Chavez VE, Livelo JB, Jimenez CL, Parrilla ML, Franzblau SG. Clinical trial of sparfloxacin for lepromatous leprosy. Antimicrob Agents Chemother 1994; 38 (1): 61 -65

42 Gelber RH, Fukuda K, Byrd S, Murray LP, Siu P, Tsang M, Rea TH. A clinical trial of minocycline in lepromatous leprosy. Brit Med J 1992; 304 (6819): $91-92$

${ }^{43}$ WHO Expert Committee on Leprosy. 7th Report. Tech Rep Ser 874 Genf: World Health Organization, 1998

${ }^{44}$ Single-Lesion Multicentre Trial Group. Efficacy of single dose multidrug therapy for treatment of single-lesion paucibacillary leprosy. Indian J Lepr 1997; 69 (2): 121 - 129

${ }^{45}$ Gupta MD. Field trials with a single dose of the combination rifampicin-ofloxacin-minocycline (ROM) for the treatment of paucibacillary leprosy. Lepr Rev 2000; 71 (Suppl.): S77-S80

${ }^{46}$ Ustianowski AP, Lockwood DNJ. Leprosy: current diagnostic and treatment approaches. Curr Opin Inf Dis 2003; 16 (5): 421 - 427

${ }^{47}$ Daumerie D. Current World Health Organization sponsored studies in the chemotherapy of leprosy. Lepr Rev 2000; 71 (Suppl.): S88 - S90

48 WHO-Study Group. Chemotherapy of leprosy for control programmes. Tech Rep Ser 675 Genf: World Health Organization, 1982

${ }^{49}$ WHO study group. Chemotherapy of leprosy. Report of a WHO study group. Tech Rep Ser 847. Genf: World Health Organization, 1994

50 WHO Expert Committee on leprosy. Tech Rep Ser 874 Genf: World Health Organization, 1998

51 Technical Advisory Group on elimination of leprosy. Report on third meeting of the WHO Conclusions and recommendations. Report No. WHO/CDS/CPE/CEE 2002.29 Genf: World Health Organization, 2002

52 Ji B, Saunderson P. Uniform MDT (U-MDT) regimen for all leprosy patients: another example of wishful thinking. Lepr Rev 2003; 74 (1): $2-6$

${ }^{53}$ World Health Organization. Guide to Eliminate Leprosy as a Public Health Problem. WHO/CDS/CPE/CEE/2000.14 Genf: World Health Organization, 2000

54 Ji B. Accompanied MDT (AMDT) - more questions than answers. Lepr Rev 2002; 73 (4): $301-307$

55 Jacobson RR. Needed research in chemotherapy of leprosy related to the individual patient. Int J Lepr Other Mycobact Dis 1996; 64 (Suppl.): S16-S20

${ }^{56}$ Curtiss R 3rd, Blower S, Cooper K, Russell D, Silverstein S, Young L. Leprosy research in the post-genome era. Lepr Rev 2001; 72 (1): 8-22

57 Ridley DS. Bacterial indices. In: Cochrane RG, Davey TF (Hrsg.). Leprosy in theory and practice. Bristol: 1964 